\title{
Concentration en métaux lourds des sédiments de l'estuaire du fleuve Comoé à Grand-Bassam (Sud-Est de la Côte d'Ivoire).
}

\author{
Keiba Noel KEUMEAN ${ }^{1}{ }^{*}{ }^{*}$, Siaka Barthélémy BAMBA², Gbombélé SORO ${ }^{1}$, Nagnin SORO $^{1}$, Bernard Soro \\ METONGO ${ }^{2}$ et Jean BIEMI 1 \\ 'Laboratoire des Sciences et Techniques de l'Eau et du Génie de l'Environnement (LSTEGE), Université Félix \\ Houphouët Boigny, 22 B.P. 801 Abidjan 22, Côte d'Ivoire. \\ ${ }^{2}$ Centre de Recherche Océanologiques (CRO), 29 rues des pêcheurs, BP V18 Abidjan, Côte d' Ivoire. \\ "Auteur correspondant, E-mail : douman2007@yahoo.fr, Tel : (225) 07211783 \\ Original submitted in on $2^{\text {nd }}$ November 2012. Published online at www.m.elewa.org on 31st January 2013.
}

\section{RESUME}

Objectifs : L'objectif de cette étude est d'évaluer l'état de la pollution en métaux lourds ( $\mathrm{Mn}, \mathrm{Ni}, \mathrm{Cr}, \mathrm{Cu}, \mathrm{Zn}$ et $\mathrm{Pb}$ ) et leurs répartitions dans les sédiments de l'estuaire du Comoé.

Méthodologie et résultats : les sédiments sont prélevés au moyen d'une benne de type Van Veen et conservés dans des sachets en plastique. La matière organique a été déterminée par perte au feu à $450^{\circ} \mathrm{C}$. Le carbonate de calcium a été dosé par calcimétrie. Les métaux ont été dosés par la méthode de la spectrométrie d'absorption atomique. L'index de géo-accumulation a montré une contamination dans l'ordre du $\mathrm{Pb}>\mathrm{Ni}>\mathrm{Cu}$. Les résultats ont également montré que les eaux de ruissellement constituent une des sources de contamination de l'estuaire et des ses sédiments.

Conclusions et applications: ces résultats pourraient servir de base aux politiques environnementales nationales et d'autres qui visent à protéger les estuaires côtiers et les organismes qui y vivent.

Mots clés : pollution, estuaire du Comoé, métaux lourds, sédiments, Côte d'Ivoire.

Heavy metal concentration ( $\mathrm{Mn}, \mathrm{Ni}, \mathrm{Cr}, \mathrm{Cu}, \mathrm{Zn}$ and $\mathrm{Pb}$ ) in sediment of the estuary of the Comoé River in Grand-Bassam (South-Eastern Côte d'Ivoire).]

ABSTRACT

Objectives: The objective of this study was to assess the state of heavy metal pollution in the sediments at the Comoé estuary and their distribution in the Comoé estuary.

Methodology and results: The sediments were sampled using a Van Veen tube and stored in plastic bags. Organic matter was determined with the method of loss on ignition at $450^{\circ}$. Calcium carbonate was determined by calcimetry. Heavy metals were determined by atomic absorption spectrometry. The geo-accumulation index showed contamination in the order of $\mathrm{Pb}>\mathrm{Ni}>\mathrm{Cu}$. The results also showed that the flowing waters constituted the source of contamination of the estuary and its sediments.

Conclusion and application: The results could serve as a basis for national and environmental policies designed to protect the coastal lagoons and their living organisms.

Key words: Pollution, Comoé estuary, heavy metals, sediments, Côte d'Ivoire. 


\section{INTRODUCTION}

La contamination des écosystèmes aquatiques par les métaux ( $\mathrm{Mn}, \mathrm{Ni}, \mathrm{Cr}, \mathrm{Cu}, \mathrm{Zn}, \mathrm{Pb}$ ) demeure un sérieux problème d'environnement de plus en plus inquiétant. Dans certains écosystèmes aquatiques, ces produits chimiques peuvent être à l'origine de la disparition de certaines espèces animales et/ou végétales et par conséquent, entrainer le dysfonctionnement de la chaine trophique (faible biodiversité...). Le cas africain est plus préoccupant à cause de la vulnérabilité des états qui manquent de moyens financiers et parfois techniques que nécessiterait la restauration d'un site aquatique pollué. Les métaux sont présents dans tous les compartiments de l'écosystème aquatique dans le monde (eau, sédiment, faune et flore) (Langstone et al ., 1999). Selon Forstner et Wittman (1981), une fraction importante des métaux lourds présents dans l'environnement aquatique est associée d'une manière réversible aux sédiments superficiels. C'est

\section{MATERIELS ET METHODES}

Présentation de la zone d'étude : Le secteur estuarien du fleuve Comoé (figure 1), objet de cette étude, est situé à l'extrémité orientale de la lagune Ebrié. Il est situé entre $5^{\circ} 12^{\prime}$ et $5^{\circ} 14^{\prime}$ de latitude Nord et $3^{\circ} 42^{\prime}$ et $3^{\circ} 44^{\prime}$ de pour ces différentes raisons qu'ils ont été étudiés par plusieurs auteurs dans le cadre des recherches sur la qualité des cours d'eau, lagunes, estuaires et lacs (Coulibaly et al ., 2010 et Chouti et al ., 2010 ; Srarfi et al ., 2011 ; Ouzair et al ., 2011 ; Obirikorang et al ., 2011). Les rares études de pollution chimiques effectuées sur l'estuaire (Soro et al ., 2009 ; Yao et al ., 2009 et Mondé et al ., 2011) n'ont été que ponctuelles, car ces auteurs se sont tous concentrés sur la lagune Ebrié d'Abidjan. A l'instar de l'estuaire de la lagune Ebrié de Côte d'Ivoire, l'estuaire du Comoé a besoin d'être scientifiquement connu afin d'éviter des catastrophes écologiques et contribuer à une protection efficace et durable. La présente étude vise à évaluer le niveau de contamination des sédiments de l'estuaire par la détermination des métaux tels que le chrome, le cuivre, le manganèse, le nickel, le plomb et le zinc.

longitude Ouest. Cette zone constitue le plus vaste estuaire du littoral ivoirien (Koffi et Abé, 1991). La zone estuarienne est alimentée par le fleuve Comoé qui draine tout l'arrière pays et couvre une superficie de $78000 \mathrm{~km}^{2}$.

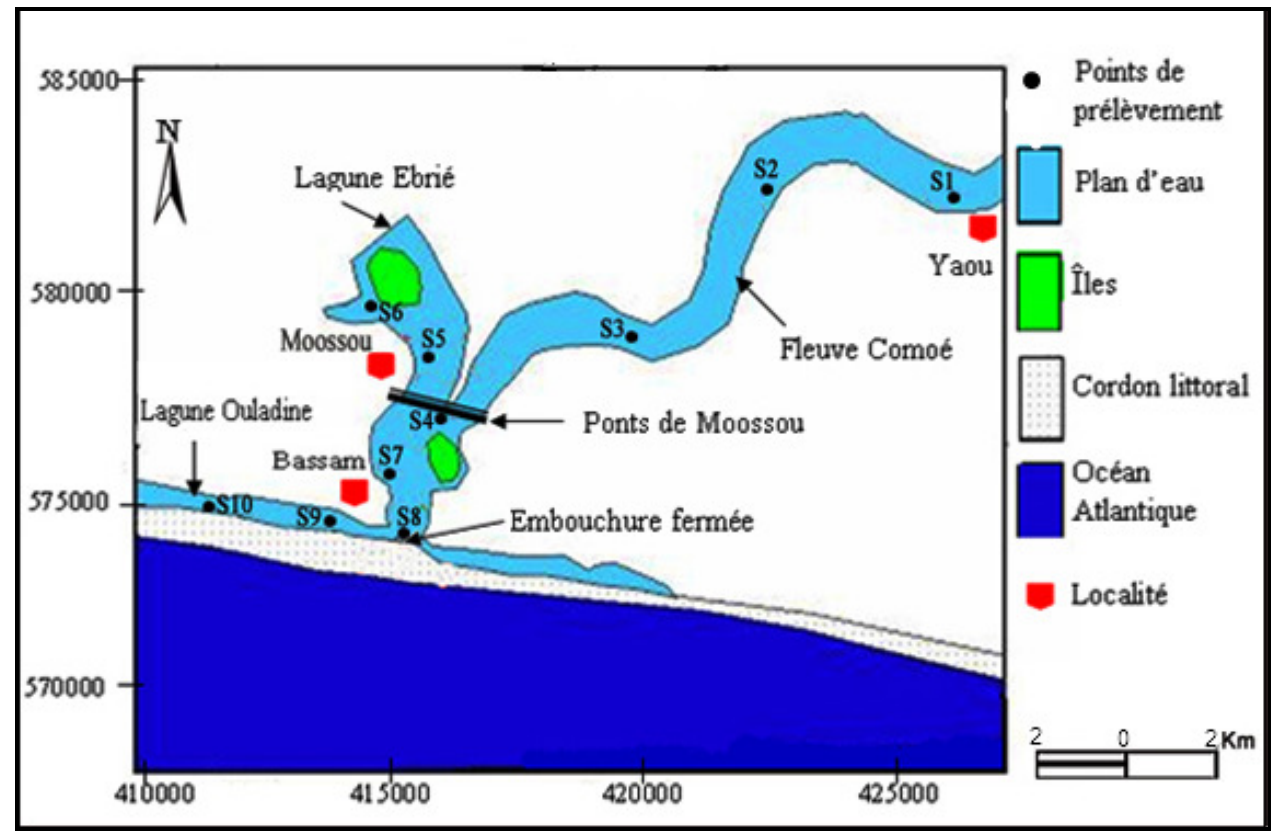

Figure 1 : Carte de localisation des sites de prélèvement sur l'estuaire du Comoé. 


\section{Keumean et al... J. Appl. Biosci. 2013. Concentration en métaux lourds des sédiments de l'estuaire du fleuve Comoé}

Echantillonnage : Huit (8) campagnes de l'estuaire du Comoé. Ces sédiments ont été conservés d'échantillonnages des sédiments de surface au moyen dans des sachets en plastiques, puis placé à l'obscurité d'une benne de type Van Veen, ont été réalisées en dix dans une glacière $\left(4^{\circ} \mathrm{C}\right)$ avant leur analyse au (10) stations (tableau 1) reparties sur tout le plan d'eau laboratoire.

Tableau 1 : Localisation géographique des sites de prélèvement dans la zone estuarienne du Comoé

\begin{tabular}{lll}
\hline & Longitude & Latitude \\
\hline Station 1 & $3^{\circ} 42^{\prime} 12.49^{\prime \prime}$ & $5^{\circ} 14^{\prime} 58.04^{\prime \prime}$ \\
Station2 & $3^{\circ} 42^{\prime} 13.71^{\prime \prime}$ & $5^{\circ} 14^{\prime} 18.42^{\prime \prime}$ \\
Station3 & $3^{\circ} 42^{\prime} 44.86^{\prime \prime}$ & $5^{\circ} 13^{\prime} 53.95^{\prime \prime}$ \\
Station4 & $3^{\circ} 43^{\prime} 12.6^{\prime \prime}$ & $5^{\circ} 13^{\prime} 00.31^{\prime \prime}$ \\
Station5 & $3^{\circ} 43^{\prime} 02.82^{\prime \prime}$ & $5^{\circ} 13^{\prime} 47.10^{\prime \prime}$ \\
Station6 & $3^{\circ} 43^{\prime} 59.41^{\prime \prime}$ & $5^{\circ} 13^{\prime} 59.27^{\prime \prime}$ \\
Station7 & $3^{\circ} 43^{\prime} 43.48^{\prime \prime}$ & $5^{\circ} 12^{\prime} 27.88^{\prime \prime}$ \\
Station8 & $3^{\circ} 43^{\prime} 26.46^{\prime \prime}$ & $5^{\circ} 11^{\prime} 51.15^{\prime \prime}$ \\
Station9 & $3^{\circ} 44^{\prime} 11.37^{\prime \prime}$ & $5^{\circ} 11^{\prime} 57.88^{\prime \prime}$ \\
Station10 & $3^{\circ} 44^{\prime} 19.49^{\prime \prime}$ & $5^{\circ} 12^{\prime} 15.59^{\prime \prime}$ \\
\hline
\end{tabular}

Analyse au laboratoire : Les métaux lourds cuivre $(\mathrm{Cu})$, chrome ( $\mathrm{Cr})$, manganèse $(\mathrm{Mn})$, nickel $(\mathrm{Ni})$, plomb $(\mathrm{Pb})$ et zinc $(\mathrm{Zn})$ ont été analysés par la méthode de la spectrométrie d'absorption atomique model VARIAN AA20. Le pourcentage de matière organique a été déterminé par perte au feu à $450^{\circ} \mathrm{C}$. Le carbonate de calcium a été dosé par calcimétrie, le taux de calcaire est calculé en fonction du volume de $\mathrm{CO}_{2}$ dégagé par action de $\mathrm{Hcl}(50 \%)$ sur le sédiment.

Traitement des données : Pour la détermination du degré de contamination, l'index de géo-accumulation Igéo (Muller, 1979) a été utilisé. Le degré de contamination a été estimé par rapport aux teneurs relatives de la croûte continentale (UCC: Upper Continental Crust) de Wedepohl (1995) (tableau 2)

Tableau 2 : Teneurs relatives de la croûte continentale selon Wedepohl (1979)

\begin{tabular}{lcccccc}
\hline & Cuivre & Chrome & Manganèse & Nickel & Plomb & Zinc \\
UCC $^{*}$ & 14 & 35 & 527 & 19 & 17 & 52 \\
\hline UCC : Upper Continental Crust & & & & &
\end{tabular}

Une étude des relations d'interdépendance a été réalisée en utilisant l'Analyse en Composantes Principales (A.C.P)

\section{RESULTATS}

Analyse de la fraction fine $(<63 \mu \mathrm{m})$, des matières organiques et des carbonates: La figure 2 présente par station les pourcentages de la fraction fine et sa teneur en matière organique. Les taux de fraction fine et dans le but de connaitre les mécanismes d'enrichissement des sédiments de l'estuaire du Comoé.

matière organique sont plus élevés à la station S8 (embouchure fermée). Les carbonates ont des teneurs élevées à la station $\mathrm{S7}$. 


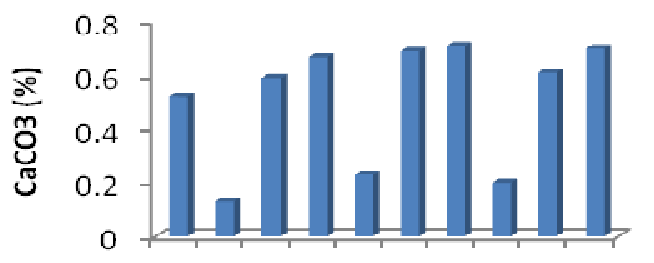 \\ ஸ त ल \\ Sites de prélèvement}

Figure 2: présente par station les pourcentages de la fraction fine et sa teneur en matière organique

Teneur en métaux des sédiments : Les résultats des analyses des éléments métalliques réalisés dans les sédiments sont présentés dans le tableau 3. Les concentrations les plus élevés sont celles du manganèse (131,95 mg.kg-1) suivi du chrome $\left(65,43 \mathrm{mg}^{-1} \mathrm{~kg}^{-1}\right)$, du nickel $\left(56,70 \mathrm{mg} \cdot \mathrm{kg}^{-1}\right)$ du plomb $\left(54,85 \mathrm{mg} . \mathrm{kg}^{-1}\right)$ du zinc $\left(49,68 \mathrm{mg} \cdot \mathrm{kg}^{-1}\right)$ et du cuivre $\left(14,77 \mathrm{mg} \cdot \mathrm{kg}^{-1}\right)$. Les stations S6, S8, S9 et S10 présentent les taux les plus élevés en métaux lourds. Le chrome, le nickel, le zinc et le manganèse suivent en général les mêmes variations saisonnières au sain d'une même station (valeurs élevés lors de la saison de crue), sauf aux stations S8 et S9 pour le chrome et $\mathrm{S} 3, \mathrm{~S} 5, \mathrm{~S} 6$ et $\mathrm{S} 7$ pour le zinc où l'évolution est différente des autres. Le plomb et le cuivre présentent également les mêmes variations saisonnières (les valeurs sont élevées lors des saisons des pluies) (figure 3 et 4 ).

Tableau 3 : Concentration (moyenne ; écart-type) des métaux lourds (mg. $\left.\mathrm{kg}^{-1}\right)$ des sédiments aux différents sites de prélèvement de 2010 à 2011

\begin{tabular}{|c|c|c|c|c|c|c|c|c|c|c|}
\hline & S1 & S2 & S3 & S4 & S5 & S6 & S7 & S8 & S9 & S10 \\
\hline & 13,17 & 14,48 & 8,77 & 15,45 & 12,29 & 21,31 & 8,91 & 14,88 & 19,32 & 19,09 \\
\hline \multirow[t]{3}{*}{$\mathrm{Cu}$} & \pm & \pm & \pm & \pm & \pm & \pm & \pm & \pm & \pm & \pm \\
\hline & 1,97 & 1,87 & 5,41 & 2,40 & 1,09 & 2,50 & 6,31 & 5,40 & 4,95 & 5,13 \\
\hline & 54,44 & 50,68 & 53,77 & 59,10 & 58,15 & 77,74 & 73,21 & 67,48 & 74,55 & 85,15 \\
\hline \multirow[t]{2}{*}{$\mathrm{Cr}$} & $\underset{36,10}{ \pm}$ & $\begin{array}{c} \pm \\
39,44\end{array}$ & $\underset{39,18}{ \pm}$ & $\begin{array}{c} \pm \\
27,39\end{array}$ & $\begin{array}{c} \pm \\
33,37\end{array}$ & $\begin{array}{c} \pm \\
29,33\end{array}$ & $\underset{26,50}{ \pm}$ & $\begin{array}{c} \pm \\
29,90\end{array}$ & $\begin{array}{r} \pm \\
7.50\end{array}$ & $\begin{array}{c} \pm \\
24,12\end{array}$ \\
\hline & 135,27 & 89,27 & 173,79 & 143,41 & 113,72 & 136,80 & 111,09 & 127,97 & 165,15 & 123,0 \\
\hline \multirow[t]{2}{*}{ Mn } & $\stackrel{ \pm}{ \pm}$ & $\begin{array}{c} \pm \\
63,40\end{array}$ & $\underset{79,39}{ \pm}$ & $\begin{array}{c}\mathbf{\pm} \\
100,39\end{array}$ & $\begin{array}{c} \pm \\
93,04\end{array}$ & $\begin{array}{c} \pm \\
58,00\end{array}$ & $\stackrel{ \pm}{ \pm}$ & $\stackrel{ \pm}{ \pm}$ & $\begin{array}{c} \pm \\
77,69\end{array}$ & $\stackrel{ \pm}{ \pm}$ \\
\hline & 47,38 & 44,80 & 54,15 & 56,37 & 51,37 & 49,78 & 57,01 & 70,25 & 62,31 & 67,46 \\
\hline \multirow[t]{2}{*}{$\mathrm{Ni}$} & $\begin{array}{c} \pm \\
28,66\end{array}$ & $\begin{array}{c} \pm \\
28,34\end{array}$ & $\begin{array}{c} \pm \\
38,64\end{array}$ & $\underset{37,31}{\mathbf{\pm}}$ & $\begin{array}{c} \pm \\
30,37\end{array}$ & $\begin{array}{c} \pm \\
27,00\end{array}$ & $\stackrel{ \pm}{ \pm}$ & $\begin{array}{c} \pm \\
38,77\end{array}$ & $\stackrel{ \pm}{ \pm}$ & $\begin{array}{c} \pm \\
39,32\end{array}$ \\
\hline & 58,62 & 53,94 & 53,55 & 47,18 & 47,84 & 71,12 & 53,43 & 53,14 & 54,18 & 55,43 \\
\hline \multirow[t]{2}{*}{$\mathrm{Pb}$} & $\begin{array}{r} \pm \\
4,97\end{array}$ & $\begin{array}{c} \pm \\
20,38\end{array}$ & $\begin{array}{r} \pm \\
3,04\end{array}$ & $\begin{array}{r} \pm \\
8,34\end{array}$ & $\begin{array}{r} \pm \\
4.21\end{array}$ & $\begin{array}{r} \pm \\
4,52\end{array}$ & $\begin{array}{r} \pm \\
3,82\end{array}$ & $\begin{array}{c} \pm \\
12,54\end{array}$ & $\begin{array}{c} \pm \\
10,18\end{array}$ & $\underset{14,12}{ \pm}$ \\
\hline & 54,94 & 45,90 & 48,58 & 42,00 & 45,00 & 59,99 & 47,02 & 52,64 & 50,84 & 50,66 \\
\hline $\mathrm{Zn}$ & $\begin{array}{c} \pm \\
18,38\end{array}$ & $\begin{array}{c} \pm \\
20,04\end{array}$ & $\stackrel{ \pm}{ \pm}$ & $\begin{array}{c} \pm \\
22,58\end{array}$ & $\begin{array}{c} \pm \\
13,33\end{array}$ & $\begin{array}{r} \pm \\
7,70\end{array}$ & $\underset{12,41}{ \pm}$ & $\begin{array}{r} \pm \\
7,70\end{array}$ & $\underset{26,51}{ \pm}$ & $\begin{array}{c} \pm \\
11,29\end{array}$ \\
\hline
\end{tabular}




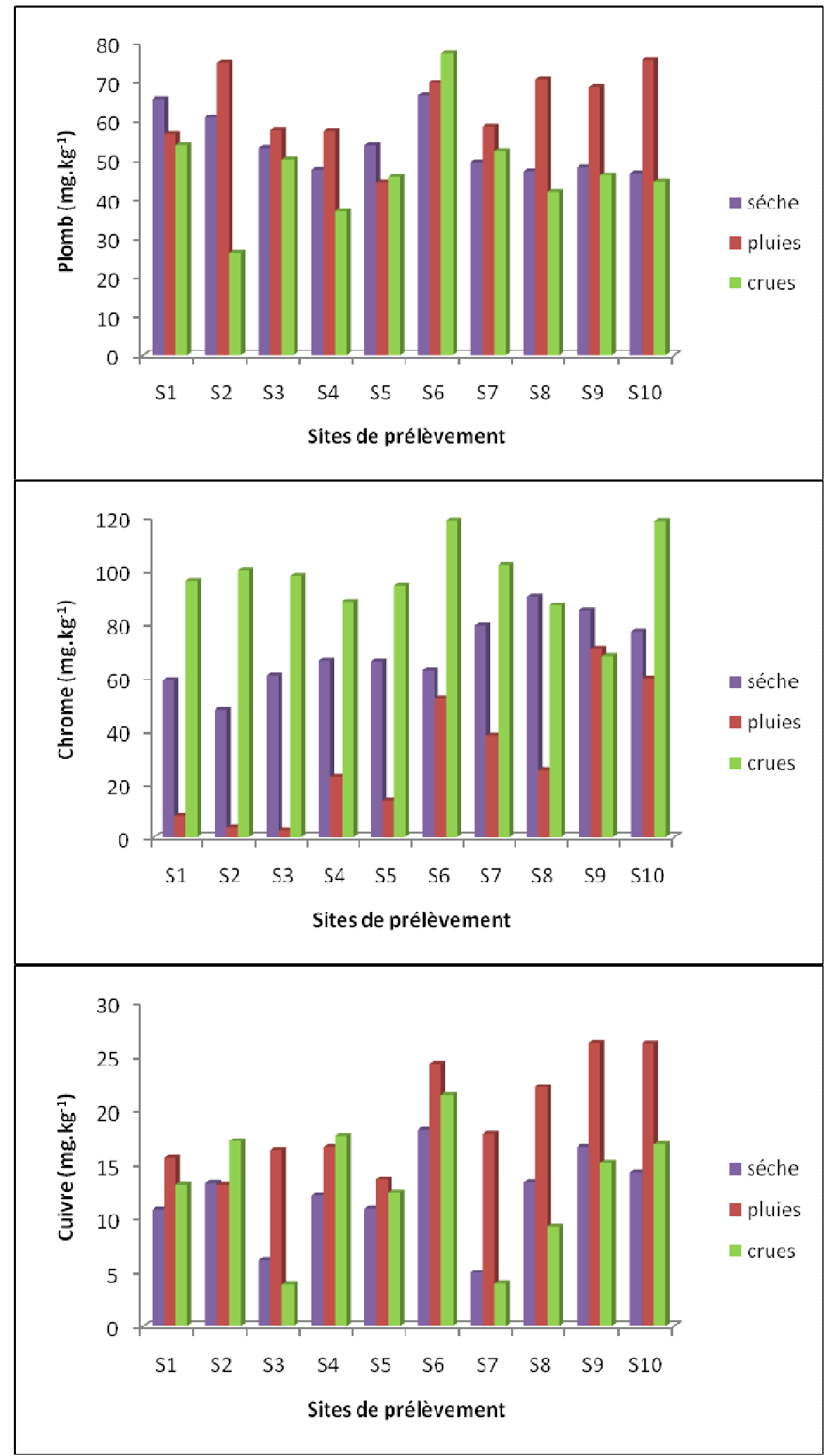

Figure 3 : Variations saisonnières de la teneur en métaux lourds des sédiments 


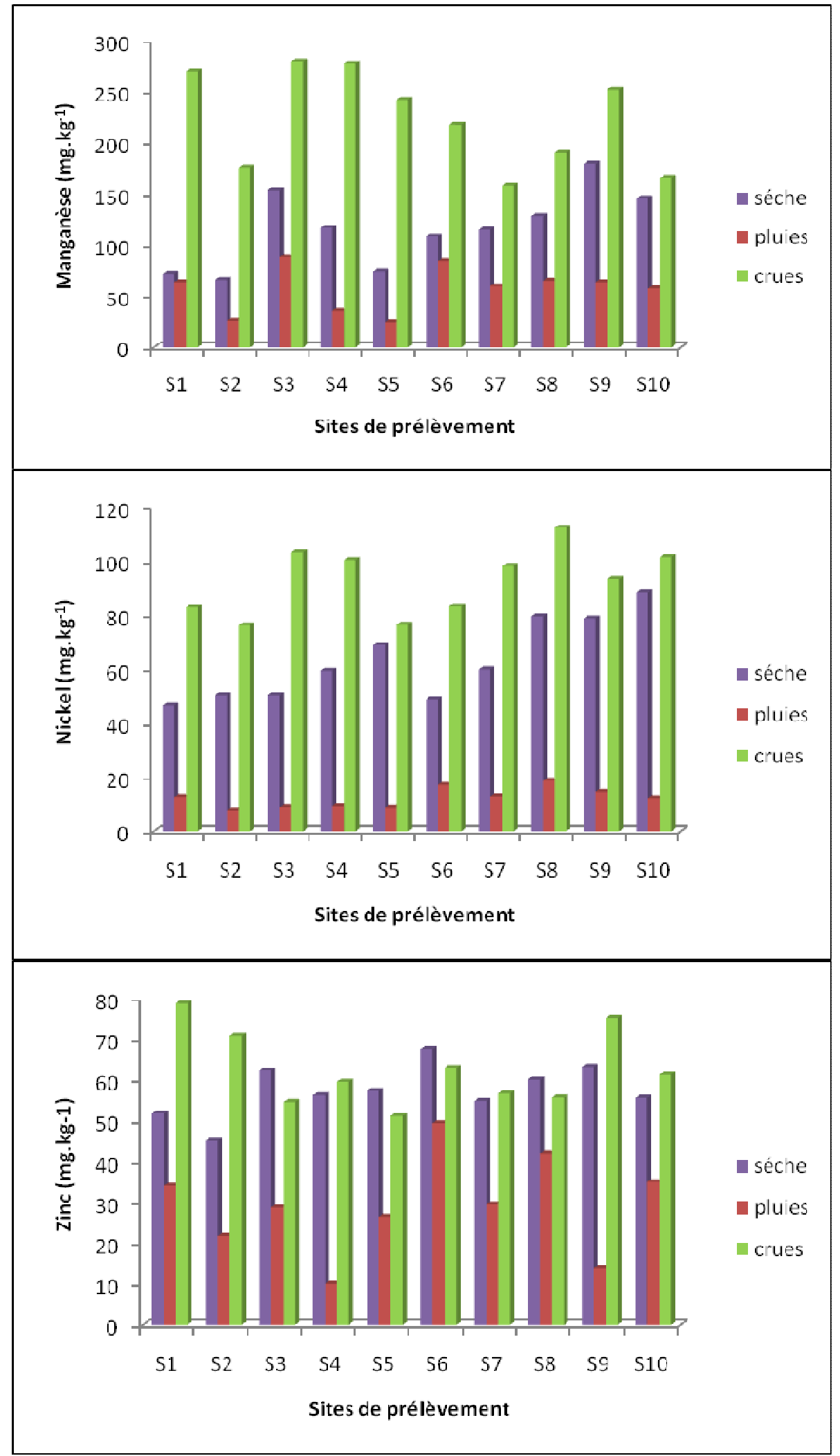

Figure 4 : Variations saisonnières de la teneur en métaux lourds des sédiments 


\section{Keumean et al... J. Appl. Biosci. 2013. Concentration en métaux lourds des sédiments de l'estuaire du}

fleuve Comoé

Index de géo-accumulation : Le tableau 4 présente les métaux analysés.

résultats de l'index de géo-accumulation des différents

Tableau 4 : Résultats de l'index de géo-accumulation des métaux étudiés

\begin{tabular}{lllllll}
\hline & Cuivre & Chrome & Manganèse & Nickel & Plomb & Zinc \\
S1 & $-0,67$ & 0,41 & $-2,54$ & 0,73 & 1,20 & $-2,54$ \\
S2 & $-0,54$ & 0,38 & $-3,15$ & 0,66 & 1,08 & $-3,15$ \\
S3 & $-1,26$ & 0,41 & $-2,9$ & 0,93 & 1,07 & $-2,19$ \\
S4 & $-0,44$ & 0,45 & $-2,46$ & 0,98 & 0,88 & $-2,46$ \\
S5 & $-0,77$ & 0,44 & $-2,80$ & 0,85 & 0,91 & $-2,80$ \\
S6 & 0,02 & 0,57 & $-2,53$ & 0,8 & 1,26 & $-2,53$ \\
S7 & $-1,23$ & 0,54 & $-2,83$ & 1,00 & 1,07 & $-2,83$ \\
S8 & $-0,50$ & 0,51 & $-2,63$ & 1,30 & 1,06 & $-2,63$ \\
S9 & $-0,12$ & 0.55 & $-2,56$ & 1,26 & 1,09 & $-2,56$ \\
S10 & $-0,13$ & 0,61 & $-2,68$ & 1,25 & 1,12 & $-2,68$ \\
\hline minimum & $\mathbf{- 1 , 2 6}$ & $\mathbf{0 , 3 8}$ & $\mathbf{- 3 , 1 5}$ & $\mathbf{0 , 6 6}$ & $\mathbf{0 , 8 9}$ & $\mathbf{- 0 , 8 9}$ \\
Maximum & $\mathbf{0 , 0 2}$ & $\mathbf{0 , 6 1}$ & $\mathbf{- 2 , 1 9}$ & $\mathbf{1 , 3 0}$ & $\mathbf{1 , 2 6}$ & $\mathbf{- 0 , 3 8}$ \\
Moyenne & $\mathbf{0 , 5 7}$ & $\mathbf{0 , 4 9}$ & $\mathbf{- 2 , 6 1}$ & $\mathbf{0 , 9 8}$ & $\mathbf{1 , 0 7}$ & $\mathbf{- 0 , 6 6}$ \\
Ecart-type & $\mathbf{0 , 4 2}$ & $\mathbf{0 , 0 7}$ & $\mathbf{0 , 2 8}$ & $\mathbf{0 , 2 2}$ & $\mathbf{0 , 1 1}$ & $\mathbf{0 , 1 5}$ \\
\hline
\end{tabular}

Manganèse : les valeurs de l'Igéo varient de $-3,43$ à 2,37 avec une moyenne de $-2,83$ traduisant une absence de pollution ;

Chrome : les valeurs de l'Igéo varient de 0,38 à 0,61 avec une moyenne de 0,49 traduisant un état de non pollué à modérément pollué ;

Nickel : les valeurs de l'Igéo varient de 0,66 à 1,30 avec une moyenne de 0,98 traduisant un état de non pollué à modérément pollué ;

Plomb : les valeurs de l'Igéo varient de 0,89 à 1,26 avec une moyenne de 1,07 traduisant un milieu modérément pollué ;

Zinc : les valeurs de l'Igéo varient de $-0,89$ à $-0,38$ avec une moyenne de $-0,66$ traduisant une absence de pollution ;

Cuivre : les valeurs de l'Igéo varient de $-1,26$ à 0,02 avec une moyenne de $-0,57$ traduisant une absence de pollution ;

Le degré de pollution, du métal le plus polluant au moins polluant suit l'ordre : $\mathrm{Pb}>\mathrm{Ni}>\mathrm{Cr}$.

Analyse en Composantes Principales (ACP): Une analyse en composante principale (ACP) réalisée à partir des données obtenues permet d'établir une corrélation entre la taille des granulats, leur association avec les métaux lourds et leur répartition aux différentes stations. Les trois premiers facteurs expriment $82,78 \%$ de la variance dont $43,58 \%$ pour $\mathrm{F} 1,23,01 \%$ pour $\mathrm{F} 2$ et $6,20 \%$ pour F3. La corrélation entre les variables et les facteurs (tableau 5) montre :

- Une bonne corrélation entre la matière organique, la fraction fine, les métaux lourds $(\mathrm{Cu}, \mathrm{Cr}$ et $\mathrm{Ni}$ ) et le facteur $\mathrm{F} 1$ et dans une moindre mesure le zinc. Le facteur F1 représente l'influence de la taille des grains sur la répartition des métaux lourds ;

- Une bonne corrélation entre le plomb, le zinc et le facteur F2. Le facteur F2 représente le facteur anthropique, car ces métaux sont associés aux apports de polluants ;

- Une bonne corrélation entre les carbonates, le manganèse et le facteur $F 3$. Cela suggère l'influence des carbonates sur la distribution du manganèse, ce métal a pour support privilégié les carbonates. 


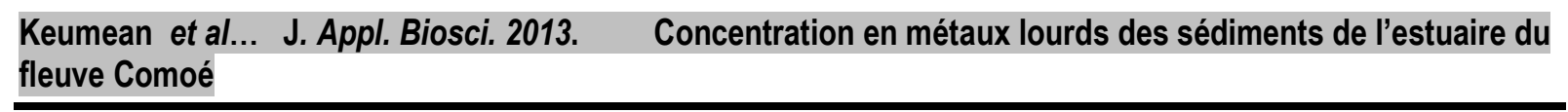

Tableau 5 : Corrélation entre les variables et les facteurs

\begin{tabular}{llll}
\hline & $\mathbf{F 1}$ & $\mathbf{F 2}$ & $\mathbf{F 3}$ \\
\hline Variance exprimée & $43,58 \%$ & $23,01 \%$ & $16,20 \%$ \\
$\mathrm{Cu}$ & 0,76 & & \\
$\mathrm{Cr}$ & 0,93 & & 0,80 \\
$\mathrm{Mn}$ & & $-0,56$ & \\
$\mathrm{Ni}$ & 0,68 & 0,83 & \\
$\mathrm{~Pb}$ & 0,47 & 0,69 & \\
$\mathrm{Zn}$ & 0,58 & & \\
$\mathrm{Ff}$ & 0,83 & $-0,49$ & 0,69 \\
$\mathrm{MO}$ & 0,75 & & \\
$\mathrm{CaCO}_{3}$ & 0,41 & & \\
\hline
\end{tabular}

$\mathrm{Ff}$ : fraction fine $(<60 \mu \mathrm{m}) ; \mathrm{MO}$ : matières organique

\section{DISCUSSION}

La répartition des éléments métalliques dans les sédiments superficiels de l'estuaire du Comoé dépend de différentes sources. Ces dernières peuvent être naturelles ou anthropiques, atmosphérique, solide ou hydrique. En étudiant l'évolution des concentrations par rapport à la localisation des échantillons, il été remarquons que les métaux étudiés ont des teneurs plus élevés dans les sédiments vaseux et fins, à l'exception du manganèse. La granulométrie est sans doute le facteur naturel, le plus important susceptible de faire varier les concentrations en métaux traces d'un sédiment. Plus la taille des grains qui composent un sédiment n'est faible, plus sa capacité à fixer les métaux présents dans le milieu est forte. De nombreux auteurs ont montré les relations existantes entre la teneur en élément métallique et la granulométrie du sédiment. Les valeurs les plus élevées se rencontrent en général dans la fraction la plus fine des sédiments. Ces mêmes constats ont été faits par Yacoub et al ., (2009) dans la lagune de Fresco en Côte d'Ivoire, Coulibaly et al ., (2009) dans les baies estuariennes d'Abidjan en Côte d'ivoire et Srarfi et al ., (2010) sur la lagune de Bizerte en Tunisie.

En ce qui concerne la matière organique, on note une teneur élevée dans la fraction fine au niveau de l'estuaire du Comoé. La relation qui apparait entre les taux en matière organique et les concentrations en métaux lourds ( $\mathrm{Ni}, \mathrm{Cr}$ et $\mathrm{Cu}$ ) s'explique par le rôle important que joue cette dernière dans la fixation et la complexation des métaux lourds dans les sédiments. Ce résultat vient corroborer les études antérieures stipulant que les métaux sont essentiellement liés à la phase organique (Yacoub et al ., 2009 ; Srarfi et al ., 2011). La distribution spatiale de la matière organique semble être étroitement liée aux faciès sédimentaires de l'estuaire. Les apports anthropiques, notamment les rejets domestiques riches en matière organique, sont d'une importance primordiale et entrent fort probablement dans la composition des sédiments superficiels de la zone d'étude.

Au niveau des carbonates, les teneurs sont reparties de manière hétérogène, et forme une association avec le manganèse. Selon Zerbe et al ., (1999) in Ouzair et al ., (2011), dans le sédiment d'un milieu aquatique, la grande partie du manganèse est liée aux carbonates. Du point de vue géochimique, les concentrations des métaux lourds dans les sédiments de l'estuaire, sujettes à des pressions anthropiques, présentent des concentrations en chrome, nickel et plomb plus élevées; ceci est confirmé par l'index de géo-accumulation du chrome, du nickel et du plomb qui montre une pollution dans l'ordre croissant $(\mathrm{Pb}>\mathrm{Ni}>\mathrm{Cr})$. Certaines concentrations en métaux des sédiments dépassent les valeurs de l'UCC estimées par Wedepohl (1995). Ces valeurs anormales peuvent être affectées à la charge polluante apportée par les différents rejets issus des activités agricoles, domestiques et artisanales. Les faibles concentrations de métaux observées en saison sèche peuvent s'expliquer par la diffusion de chaque élément dans la phase dissoute, en raison des conditions physico-chimiques (température élevée) et le relargage du matériel particulaire sous l'action des courants. Les faibles concentrations pendant la saison des pluies pourraient s'expliquer certainement par les phénomènes de dilution qui s'opèrent par des apports de sédiments moins ou non contaminés (Yao et al ., 2009).

Par contre pour les concentrations élevées en plomb, les sédiments de l'estuaire se seraient contaminés directement par les retombées atmosphériques ou de manière indirecte après lessivage des routes par les eaux de pluies. Une accumulation non négligeable en plomb peut être en relation avec le trafic routier du périmètre 
urbain. Ces métaux sont lessivés par les eaux pluviales pour enrichir les eaux usées en ces éléments (Ouzair et al ., 2011). Les teneurs élevées en nickel et chrome témoignent d'une pollution de l'estuaire par ces éléments, mais le manque d'industrie dans la ville de GrandBassam, nous fait dire que cette pollution serait la conséquence des rejets urbains sans traitement, du lessivage des dépôts incontrôlés au bord de l'estuaire et des activités de garage dans la ville (Yao et al ., 2009).

\section{CONCLUSION}

Les résultats obtenus dans ce travail nous ont permis de faire une évaluation de la contamination en métaux lourds dans les sédiments de l'estuaire du fleuve Comoé. Les teneurs sont plus élevées soit lors des saisons des crues et sèche, soit pendant les saisons des crues et des pluies selon la station ou le métal. Ceci en relation avec les conditions physico-chimiques qui contrôlent le relargage des métaux et la composition métallique des sédiments

\section{REFERENCES}

Chouti W, Mama D, Changotade O, Alapini F, Boukari M, 2010. Etude des éléments traces métalliques contenus dans les sédiments de la lagune de Porto-Novo (Sud Bénin). Journal of Applied Biosciences 34: 2186-2197.

Coulibaly AS, Mondé S, Wognin VA, Aka K, 2010. Dynamique des éléments traces métalliques dans les sédiments des baies d'Abidjan (Baie du Banco et Rade Portuaire). European Journal of Scientific Research 46 (2) : 204-215.

Coulibaly AS, Mondé S, Wognin VA, Aka K, 2009. Analyse des éléments traces métalliques (ETM) dans les baies estuariennes d'Abidjan en Côte d'ivoire. Afrique sciences 05 (3) : 77-96.

Förstner U et Wittman GTW, 1981. Metal pollution in the Aquatic Environment ( $\left({ }^{\text {nd }} e d n\right)$. Springer-Verlag: Berlin; 486.

Koffi K. et Abe J, 1991. Contribution à l'étude des modifications hydro-sédimentaires consécutives à la réouverture artificielle de l'embouchure du Comoé à Grand-Bassam. Journal Ivoirien d'Océanologie et Limnologie, 1(2) : 47-60.

Langstone WJ, Burt GR, Pope ND, 1999. Estuarine, coastal and shelf science, 48: 519-540.

Mondé S, Coulibaly AS, Wango T, Aka K, 2011. Simulation predictive de la qualité des eaux lors des rejets anthropiques dans la lagune Ebrié (Côte d'Ivoire). Inter. J.Of African Studies, 4: 2840.
Les concentrations en métaux lourds obtenues au niveau des sédiments de surface de l'estuaire du Comoé sont inférieures à celles mesurées dans les sédiments des baies d'Abidjan (Coulibaly et al ., 2010), l'estuaire de la volta (Obirikorang et al ., 2011), la lagune de Porto-Novo (Chouti et al ., 2010) respectivement en Côte d'Ivoire, Ghana et Bénin.

apportés en estuaire. L'index de géo-accumulation a montré une contamination des sédiments dans l'ordre $(\mathrm{Pb}>\mathrm{Ni}>\mathrm{Cr}$ ). Le niveau de contamination et la croissance de la teneur des sédiments en métaux lourds de la zone urbaine de l'estuaire soulignent l'urgence de diminuer les rejets de polluants dans ces milieux, ce qui éviterait les problèmes de santé publique, de disparation des ressources halieutiques et de l'aquaculture.

Muller G, 1979. Schwermetalle in den sedimen der Rheins-veränderungen seit 1971. Umschau, 79(24): 778-783.

Obirikorang KA, Madkour HA, Amisah S, Adjei-Boateng D, Otchere FA, 2001. Heavy metal accumulation $(\mathrm{Mn}, \mathrm{Zn}, \mathrm{Fe}$ and $\mathrm{Hg}$ ) in the surface sediments of the Volta estuary, Ghana. Australian J. of Basic and Appl. Sci. 5(9): 564-570.

Ouzair A, Lebkiri A, Belghyti D, Fadli M, Rifi El H, Hbaiz El M, Ouihman El M, Lebkiri M, 2011. Etude typologique de la contamination métallique du sédiment et certaines caractéristiques physicochimiques de l'eau d'Oued Rdom (Maroc). SciencesLib Editions Mersenne 3(110403).

Soro G, Soro MB, Soro N, Ahoussi KE, Kouamé KF, Zade SGP, Soro T, 2009. Métaux lourds ( $\mathrm{Cu}$, $\mathrm{Cr}, \mathrm{Mn}$ et $\mathrm{Zn}$ ) dans les sédiments de surface d'une lagune tropicale africaine: cas de la lagune Ebrié (Côte d'Ivoire). Int. J. Biol. Chem. Sci. 3(6) : 1408-1427.

Srarfi F, Tagourti M A, Tlig S, Slim Shimi N, 2010. Ifluence de la separation granulométrique sur la composition des sédiments en métaux lourds de la lagune de bizerte (Tunisie). Communication sciences et technologie, $\mathrm{N}^{\circ} 8$ janvier 2010.

Srarfi F, Tagourti M A, Tlig S, Slim Shimi N, 2011. Tinja : un chenal de transition hydrologique et géochimique entre lagune de Bizerte et Garaet Ichkeul (NE Tunisien). Larhyss Journal, (9) : 7382. 
Wedepohl KH. 1995. The composition of continental crust. Goechimica and cosmochimica Acta. 59(7): 1217-1232.

Yacoub I, Kouassi AM, Adingra AA, Biemi J, 2009. Concentration en métaux lourds des sédiments d'une lagune côtière tropicale: lagune de Fresco (Côte d'Ivoire). J.Appl. Biosci. 18: 10091018.

Yao KM, Soro MB, Trokourey A, Bokra Y, 2009. Assessment of sediments contamination by heavy metals in a tropical lagoon Urban Area (Ebrié lagoon, Cote d'Ivoire). Eur. J. of Sci. Res. 34(2): 280-289. 